Check for updates

Cite this: RSC Adv., 2018, 8, 14109

\title{
In silico analysis and in vivo tests of the tuna dark muscle hydrolysate anti-oxidation effect $\uparrow$
}

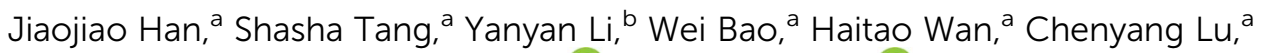 \\ Jun Zhou, ${ }^{* a}$ Ye Li, ${ }^{a}$ Lingzhi Cheong (D ${ }^{a}$ and Xiurong Su (D) *a
}

Hydrolysate is a mixture of various peptides with specific functions. However, functional identification of hydrolysate with high throughput is still a difficult task. Furthermore, using in vivo tests via animal or cell experiments is time and labor-intensive. In this study, the peptides component of hydrolysate derived from the tuna dark muscle was measured via MALDI-TOF/TOF-MS, and the functions of the KEFT (LysGlu-Phe-Thr), EEASA (Glu-Glu-Ala-Ser-Ala) and RYDD (Arg-Tyr-Asp-Asp) peptides, which were found with the highest proportion, were predicted via Discovery Studio 2016 software. All three peptides were predicted to bind to the Keap1 protein with the highest fit-value and to affect the activity of Keap1, which is involved in anti-oxidation pathways. Subsequently, mice experiments showed that administration of tuna dark muscle hydrolysate increased the levels of superoxide dismutase and glutathione peroxidase in the serum and liver $(P<0.05)$ and decreased the malondialdehyde level $(P<$ $0.05)$ as well as transcription of Keap1 $(P>0.05)$, which are consistent with the in silico analysis results using Discovery Studio 2016 software. The combination of in silico analysis and in vivo tests provided an alternative strategy for identifying hydrolysate function and provided insight into high-value utilization of protein hydrolysate.

Received 29th January 2018

Accepted 6th April 2018

DOI: $10.1039 / \mathrm{c} 8 \mathrm{ra00889b}$

rsc.li/rsc-advances and were evaluated according to their 2,2-diphenyl-1picrylhydrazyl radical scavenging activity, reducing power and metal chelating activity. In addition, two types of peptides extracted from the Rastrineobola argentea via different enzyme treatments were shown to have strong antioxidant activities of $31.5 \%$ and $49.5 \%$ by using oleic acid (lipid) peroxidation inhibition to measure their antioxidant potential. Meanwhile, the corresponding data for the commercial antioxidant butylhydroxytoluene is $29 \%{ }^{4}$

Tuna belongs to the Osteichthyes, Perciformes, and Scombridae classes and is a highly migratory fish that lives in the middle of bodies of water. Tuna is well appreciated worldwide because of its high nutritional value and potential health benefits. ${ }^{5}$ However, large quantities of fish protein (especially the dark muscle, $50-70 \%$ of the raw material) are regarded as by-products and are discarded without any attempt at recovery or valorization. Studies have shown that tuna peptides have various functions. Peptides isolated from the tuna backbone protein have strong antioxidant activity, ${ }^{6}$ and peptides obtained from the tuna cooking juice have angiotensin I-converting enzyme (ACE) inhibition activity that can be used to lower blood pressure. ${ }^{7}$ Dark muscle contains abundant protein and is a promising and potential alternative protein source for the preparation of peptides. However, the enzymatic hydrolysate is a mixture of various peptides, and functional identification of the hydrolysate with high throughput still a hard task, whereas
${ }^{a}$ School of Marine Science, Ningbo University, 818 Fenghua Road, Ningbo, China. E-mail: suxiurong_public@163.com; zhoujun1@nbu.edu.cn; Fax: +86 574 87608368; Tel: +8657487608368

${ }^{b}$ College of Agriculture and Life Sciences, Cornell University, Ithaca, USA

$\dagger$ Electronic supplementary information (ESI) available. See DOI: $10.1039 / \mathrm{c} 8 \mathrm{ra} 00889 \mathrm{~b}$ 
one-by-one in vivo tests via animal or cell experiments are time and labor-intensive.

Discovery Studio 2016 (DS 2016) is a new generation of molecular modeling software that is mainly used in protein structure and drug discovery. ${ }^{8}$ Reverse docking is a method that does the opposite of virtual screening by using docking. ${ }^{9}$ The concept of one-ligand with many targets is an important tool for the drug development. ${ }^{10}$ Molecular docking is based on geometric matching and energy matching to find the best match mode between receptors and ligands. ${ }^{11}$ The DS3.1 LigandFit and CDOCKER docking programs were used to obtain a preliminarily estimate of the binding sites of $\alpha$-cyperone to human serum albumin. ${ }^{8}$ To explore the structural-functional mechanism between purified peptides (Tyr-Arg and Ile-Arg) and ACE, DS3.0 was used to simulate computational molecular docking. ${ }^{12}$ Recently, traditional cholesterol-lowering statins were predicted to have anti-tumor activity via Discovery Studio software, and this prediction was subsequently confirmed by cell experiments. The main ingredients, ginseng and ginsenosides, were evaluated for anti-cancer properties via reverse docking. ${ }^{13}$ In addition, ganoderma secondary metabolites were found to have antiviral activity by in silico profiling, ${ }^{14}$ and the simulation results were verified by in vitro experiments. Previous studies indicated the peptides can be used as ligands in the Discovery Studio, ${ }^{15}$ and the target proteins binding to the peptide ligands might provide information about the function of the peptides, as well as the hydrolysate (peptides mixture).

In consideration that the traditional experiment-and-screen methods suffer from being time and labor-intensive, a strategy combining in silico analysis and in vivo tests to screen the functions of hydrolysate with high throughput is provided in this study. After the determination of optimal enzymatic hydrolysis conditions with single factor and response surface experiments, the majority peptides in the hydrolysate are measured, the functions are subsequently predicted via software Discovery Studio 2016, and further verified via animal experiments.

\section{Materials and methods}

\section{Optimization of enzymatic hydrolysis conditions}

As previously described, the optimal enzymatic hydrolysis conditions were determined via single factor and response surface experiments, including the combination of the enzyme type, hydrolysis temperature, hydrolysis time, and enzyme and solid-liquid ratio content. ${ }^{16}$ The detailed process for optimizing the enzymatic hydrolysis conditions is described in the ESI. $\dagger$

\section{Peptide identification}

Hydrolysation of tuna dark muscle (Ningbo Today Food Co., Ltd, Ningbo, China) was performed under optimal conditions as described previously. After 10 min inactivation with boiling water, it was cooled to room temperature and the solution was centrifuged at $4000 \mathrm{rpm}$ at $4{ }^{\circ} \mathrm{C}$ for $10 \mathrm{~min}$ to obtain a precipitate (Thermo Scientific, MA, USA). Then, polypeptides were separated by ultrafiltration. The low molecular weight polypeptides were obtained using ultrafiltration membrane Millipore 8050 (Millipore, MA, USA) and stored in a vacuum freeze-drying machine (Free Zone 2.5 L, LABCONCO Co., Ltd, Kansas City, USA) for $24 \mathrm{~h}$.

As previously described, the hydrolysate was resuspended in $60 \mu \mathrm{L}$ of methanol/water $(50 / 50, \mathrm{v} / \mathrm{v})$ with $0.2 \%$ formic acid and $2 \mathrm{mM}$ sodium acetate prior to matrix-assisted laser dissociation time-of-flight mass spectrometer analysis ${ }^{17}$ (MALDI-TOF/TOF AB SCIEX, Applied Biosystems, CA, USA). The sample $(0.5 \mathrm{~mL})$ was directly spotted on a MALDI plate and mixed with an equal volume of 2.5 dihydroxybenzoic acid-matrix prepared by suspending $10 \mathrm{mg}$ of 2.5 dihydroxybenzoic acid in $1 \mathrm{~mL}$ of a water/ methanol $(50 / 50, \mathrm{v} / \mathrm{v})$ solution containing $1 \mathrm{mmol} \mathrm{l}^{-1}$ sodium acetate. The MALDI plate was dried under vacuum to ensure uniform crystallization. Mass spectra were acquired using a 5800 MALDI TOF/TOF Analyzer (AB SCIEX, Applied Biosystems, CA, USA).$^{18}$ The samples were collected and analyzed by MASCOT ${ }^{19}$ and NCBI to obtain the structure of the polypeptide. The precise molecular weight was determined by primary mass spectrometry, and the amino acid sequence was determined by secondary mass spectrometry. ${ }^{20}$

\section{Reverse molecular docking based on ligands}

The ligand Profiler and CDOCKER docking programs implemented in Discovery Studio 2016 software (Beijing Chong Teng Technology Co., Ltd, Beijing, China) were used in this study. First, the structures of the peptides were constructed using Discovery Studio 2016 software. The structure was energy minimized using the steepest descent and conjugate gradient techniques. Then, the "Prepare Ligands" program in Discovery Studio was used to generate multiple conformations. This process was performed using the DS Ligand Profiler tool. In this work, we used the Pharma DB as our target database. During the procedure, the parameters were set to the shape of PharmaDB pharmacophores, to the most selective of the input PharmaDB pharmacophores, and to be true for save aligned ligands. The other parameters were set to the default values.

Pharmacophore crystal structures with fit-value $>3$ were obtained from the protein data bank (PDB) for docking simulations. The procedure was performed using the CDOCKER tool. During the procedure, the proteins were downloaded from the PDB as receptors, and the peptides were set as ligands. The ligand-receptor complex energy scores determined whether the ligands and receptor proteins bonded.

\section{Animal experiment design}

All of the experimental procedures and animal care were performed in accordance with the Guide for the Care and Use of Laboratory Animals prepared by the Ningbo University Laboratory Animal Center (affiliated with the Zhejiang Laboratory Animal Common Service Platform), and all of the animal protocols were approved by the Ningbo University Laboratory Animal Center under permit number no. SYXK (ZHE 20080110).

After 2 weeks of acclimatization, twenty-four 10 week-old male ICR mice $(23.5 \pm 2.3 \mathrm{~g}$, Laboratory Animal Center of 
Table 1 Sequence of primers used in the qRT-PCR analysis

\begin{tabular}{lll}
\hline Gene name & Primer sequence & Product size \\
\hline Prkag2 & F:5'-GCAGAAAAACTACAGCAGAAGACTC-3' & \multirow{2}{*}{$123 \mathrm{bp}$} \\
& R:5'-CTTGCAACGTAGTGTCGAAGAC-3' & \\
Keap1 & F: 5'-CTGGTATCTGAAACCCGTCTA-3' & $117 \mathrm{bp}$ \\
& R:5'-TGGCTTCTAATGCCCTGA-3' & \\
3-Actin & F: 5'-CTGTCCCTGTATGCCTCTG-3' & $218 \mathrm{bp}$ \\
& R:5'-ATGTCACGCACGATTTCC-3' & \\
& &
\end{tabular}

Zhejiang province, Zhejiang, China, SCXK (Zhejiang) 2014-0001 no. 1605200003) were randomly divided into a control group and an experimental group. The mice in the experimental group were gavaged with $300 \mathrm{mg} \mathrm{kg}{ }^{-1} \mathrm{~d}^{-1}$ tuna dark muscle hydrolysate ${ }^{21}$ and the mice in the control group were given the same amount of normal saline. The twelve mice in each group were housed in three cages (four per cage). During the experiment, mice had free access to water and feed, and room temperature was maintained at $23-25{ }^{\circ} \mathrm{C}$; night and day intervals were set to $12 \mathrm{~h}$.
Body weight was measured every three days. After 40 days of feeding, all animals were anaesthetized by enflurane. Then, blood samples were taken from the orbital plexus. We separated serum via centrifugation for $15 \mathrm{~min}$ at $4{ }^{\circ} \mathrm{C}$ at $3000 \mathrm{rpm}$ and stored it at $-80^{\circ} \mathrm{C}$. Next, all animals were sacrificed by cervical dislocation. For further analysis, tissues, including the liver and brain, were weighed, and immediately frozen in $-80{ }^{\circ} \mathrm{C}$.

\section{Physiological and biochemical indexes}

Biochemical measurements of TC (total cholesterol), TG (triglyceride), HDL (high-density lipoprotein), LDL (low density lipoprotein), SOD (superoxide dismutase), GSH-Px (glutathione peroxidase) and MDA (malondialdehyde) were measured via kits (Nanjing Jiancheng Bioengineering Institute, Nanjing, China) according to the manufacturer's procedure.

\section{Quantitative real-time PCR}

As previously described, ${ }^{22}$ total RNA from frozen liver and brain was extracted using TransZol Up Plus RNA Kit (TRAN, Beijing, China). Residual genomic DNA was removed using RNase-free

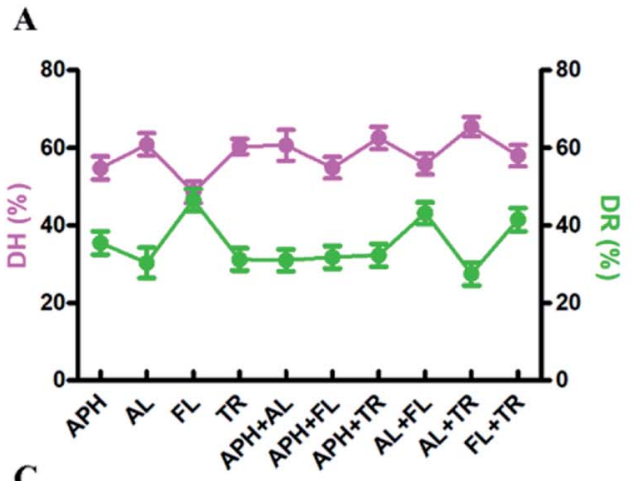

B
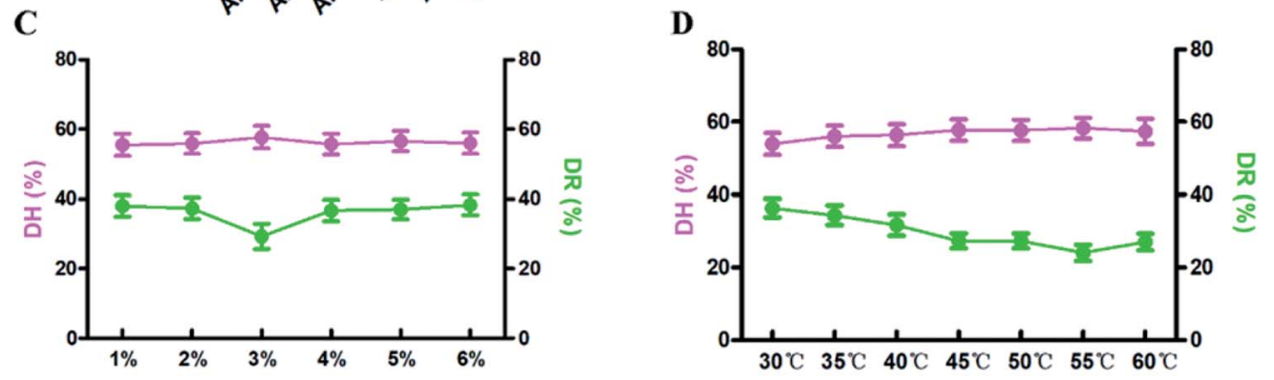

E
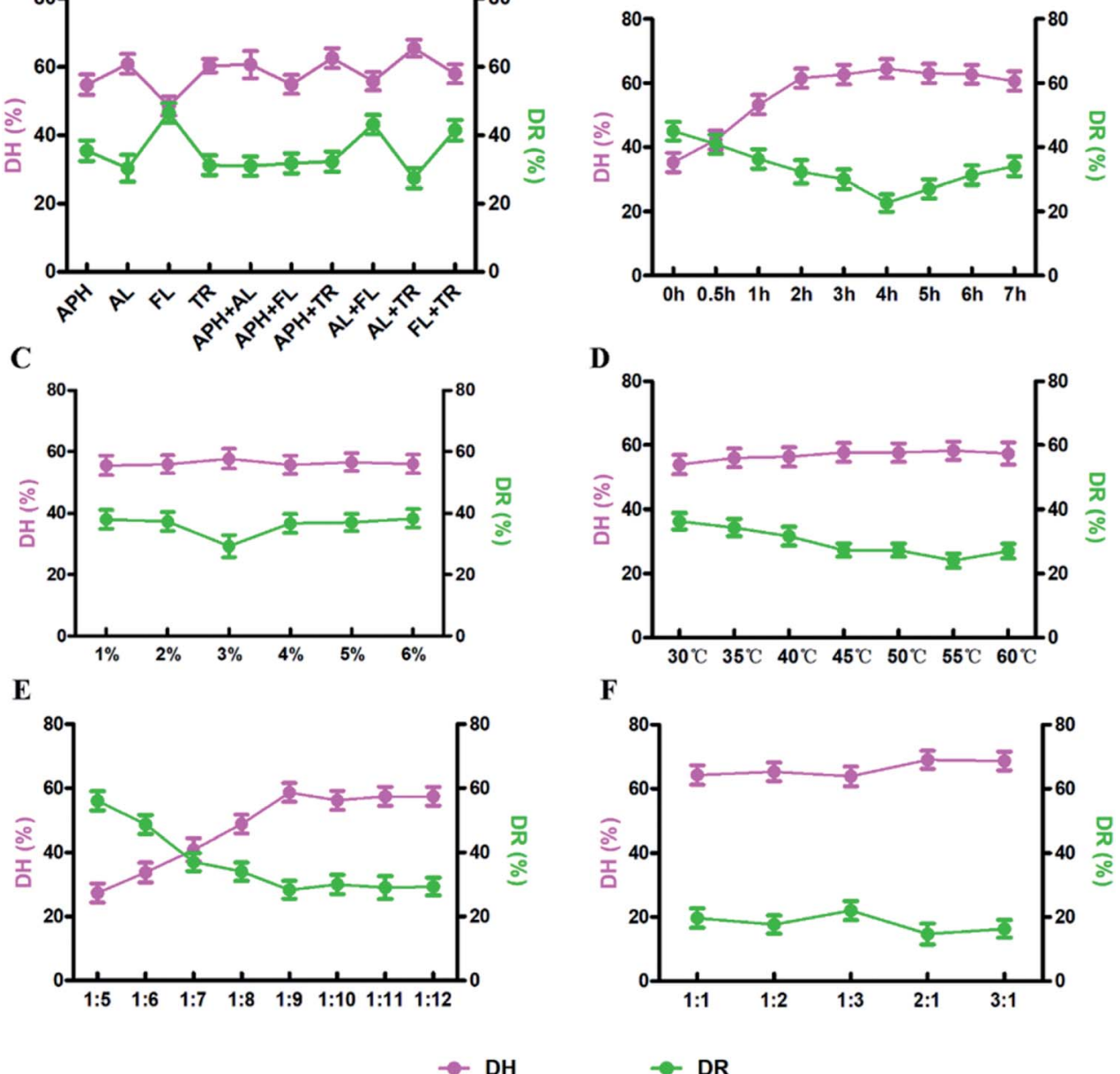

Fig. 1 Effects of the different factors on enzymatic treatment. (A) Types of enzyme (APH: animal protein hydrolase, AL: alkaline protease, FL: flavourzyme, TR: trypsin), (B) time, (C) enzyme concentration, (D) temperature, (E) solid-to-liquid ratio, (F) enzyme ratio. 

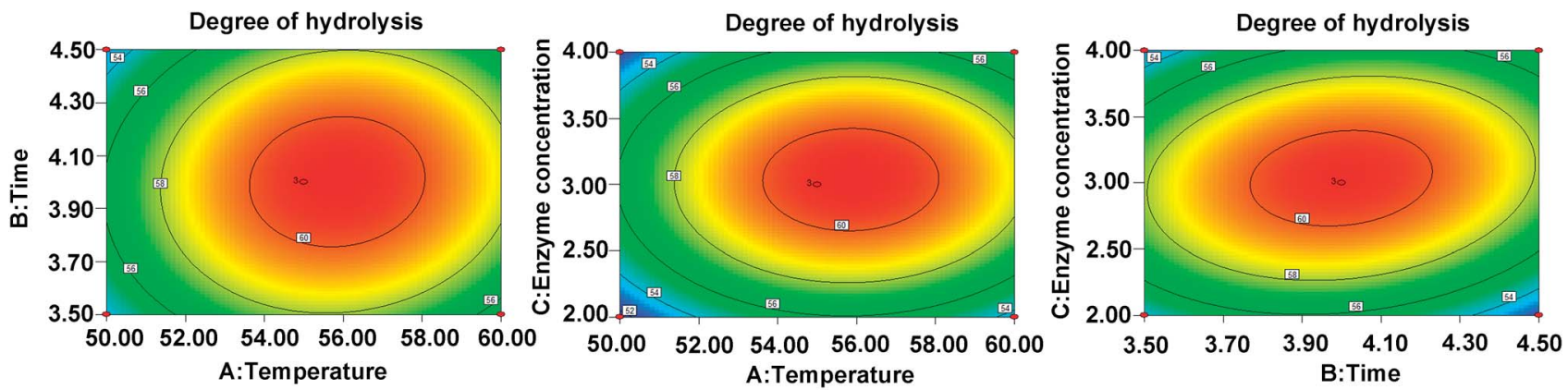

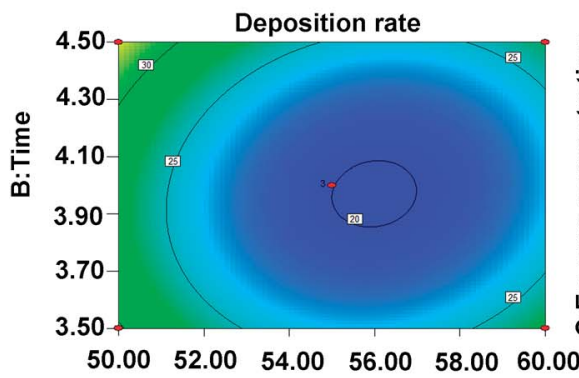

A:Temperature
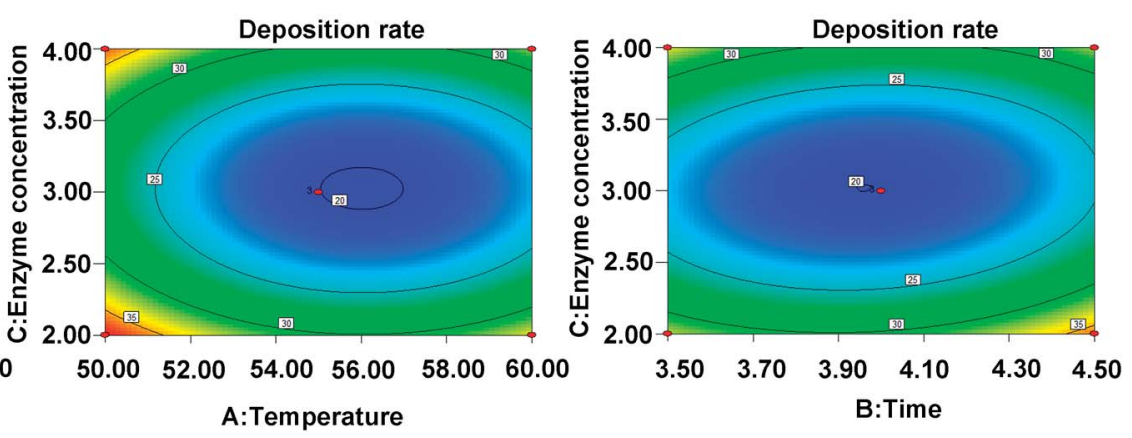

Fig. 2 The contour plots demonstrating the effects of temperature (A), enzymolysis time (B) and enzyme concentration (C) on the DH (Y1) and DR (Y2).

DNase I (MBI Fermentas, Vilnius, Republic of Lithuania) according to the manufacturer's protocol. The RNA concentration was determined using a NanoDrop 2000 UV-Vis spectrophotometer (Thermo Scientific, MA, USA). Subsequently, reverse transcription was achieved using total RNA as the starting material and the TransScript All-in-One First-Stand cDNA kit (TRAN, Beijing, China). The transcriptional levels of genes were determined by quantitative real-time RT-PCR (qRTPCR) performed on a Rotor-Gene 6000 realtime PCR machine (Corbett, Australia) with SYBR ${ }^{\circledR}$ Premix Ex TaqTM II according to the manufacturer's protocols. ${ }^{22}$ The samples were run at $95{ }^{\circ} \mathrm{C}$ for $5 \mathrm{~min}$, followed by 40 cycles of $15 \mathrm{~s}$ denaturation at $95{ }^{\circ} \mathrm{C}, 30 \mathrm{~s}$ annealing at $55^{\circ} \mathrm{C}$ and elongation for $30 \mathrm{~s}$ at $72{ }^{\circ} \mathrm{C}$, followed by melting curve analysis.

Target genes were selected based on Discovery Studio software predictions. The primers were designed using software Primer 3, with the amplification efficiency of the target and reference genes certified using the same program. The relative expression levels of the target genes were normalized internally to the 16S rDNA level and quantified using the $2^{-\Delta \Delta \mathrm{CT}}$ method with $\beta$-actin as the housekeeping gene. ${ }^{23}$ For each gene, the reference samples were taken as having an expression level of 1.0, and the data for other samples were expressed as the fold increase of the mRNA level over the reference sample. The sequences of the primer pairs are listed in Table 1.

\section{Statistical analysis}

All statistical analyses were performed using the SPSS10.0 (SPSS, Inc., Chicago, USA) statistical package. $P<0.05$ was considered statistically significant. All results are presented as the mean \pm standard deviation (S.D.).

\section{Results}

Optimization of the enzymatic hydrolysis conditions

$\mathrm{DH}$ and DR indexes are used as the indexes to measure the enzymatic hydrolysis efficiency, the combination with the highest DH and the lowest DR is regarded as the most efficient one (Fig. 1). In this study, the optimal enzymolysis condition is confirmed via single factor experiment (Fig. 1) and response surface experiment (Fig. 2, ESI Tables S1 and S2 $\dagger$ ), that is trypsin and alkaline combination at the ratio of $2: 1,4 \mathrm{~h}$ hydrolysis under $55{ }^{\circ} \mathrm{C}$, the enzyme concentration was $3 \%$ and the solid-liquid ratio was $1: 9$. In this condition, the $\mathrm{DH}$ and DR reached $60.7 \%$ and $19.8 \%$, respectively.

\section{Polypeptide composition in hydrolysate}

The amino acid sequence of the enzymatic hydrolysate was determined by MALDI TOF/TOF-MS. In the $m / z$ 0-2000 range, 403 peaks were detected, and three peaks showed higher intensities than the others, corresponding to $\mathrm{m} / \mathrm{z} 506.1051, \mathrm{~m} / \mathrm{z}$ 524.1039 and $m / z 568.0896$ (ESI Table S3 $\dagger$ ), which were predicted to be KEFT (Lys-Glu-Phe-Thr), EEASA (Glu-Glu-Ala-SerAla) and RYDD (Arg-Tyr-Asp-Asp) (Fig. 3).

\section{Polypeptide function prediction using Discovery Studio software}

The potential activity of the three polypeptides was screened via the Discovery Studio software reverse search function, and the results are shown in Table 2. KEFT can bind to 5dad (Keap1) (3.56 fit-value), 1uk4 (replicase polyprotein) (3.35 fit-value) and $1 \mathrm{~m} 4 \mathrm{~d}$ (aminoglycoside $2^{\prime}-N$-acetyltransferase) (3.25 fit-value); 

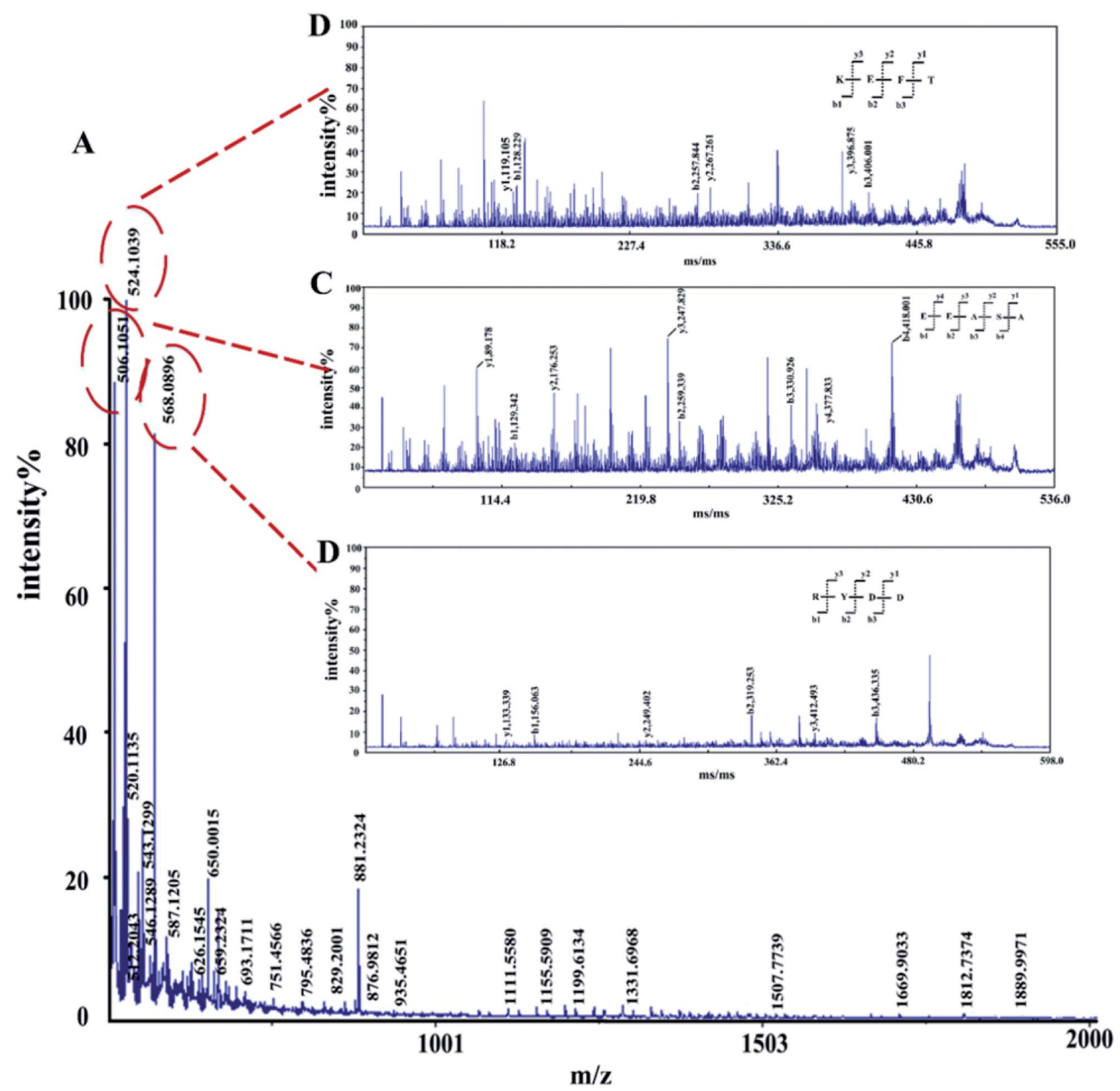

Fig. 3 MALDI-TOF/TOF tandem mass spectra of polypeptides. (A) First-order mass spectra of the enzymatic peptides. (B)-(D) Secondary mass spectrometry.

EEASA can bind to 5 dad (3.69 fit-value), 1 uk4 (3.60 fit-value) and 1086 (angiotensin-converting enzyme) (3.33 fit-value); and RYDD can bind to 5 dad (3.62 fit-value), 1jj7 (antigen peptide transporter1) (3.41 fit-value) and 1uk4 (3.37 fit-value). Keap1 is an important regulator of cellular oxidative stress, and all three domain peptides can bind to Keap1 with the highest fit-value. Therefore, we presumed that Keap1 is a potential target.

To explain how the three polypeptides bind to the Keap1 protein, the molecular docking function in the Discovery Studio software was used. The molecular docking results should be based on the receptor active site, receptor-ligand-CDOCKER interaction energy (CIE), number of interacting amino acid residues and other information to determine the extent of binding $^{24}$ (Table 3). Previous studies have reported that TX6 binds to the Keap1 protein to inhibit the activity of Keap1 as well as the Nrf2/ARE signaling pathway. ${ }^{25}$ Therefore, we chose TX6 as a reference to evaluate the degree of peptide and Keap1 protein binding, and the CIE values of KEFT, EEASA and RYDD were 66.6, 76.2 and 71.3, respectively, while the CIE value for the reference TX6 was 26.1. TX6 and the peptides KEFT, EEASA and RYDD have similar docking sites that are located at the same active site (Fig. 4A, D, G and J). The molecular docking study showed that TX6 established $10 \pi$ bonds with 5 Keap 1 amino acid residues, and the remaining seven amino acid residues interacted with Keap1 by VDW (Fig. 4B and C). There are 14 amino acid residues that interact with KEFT and Keap1, and the 
Table 2 The results of potential targets screening with high fit values

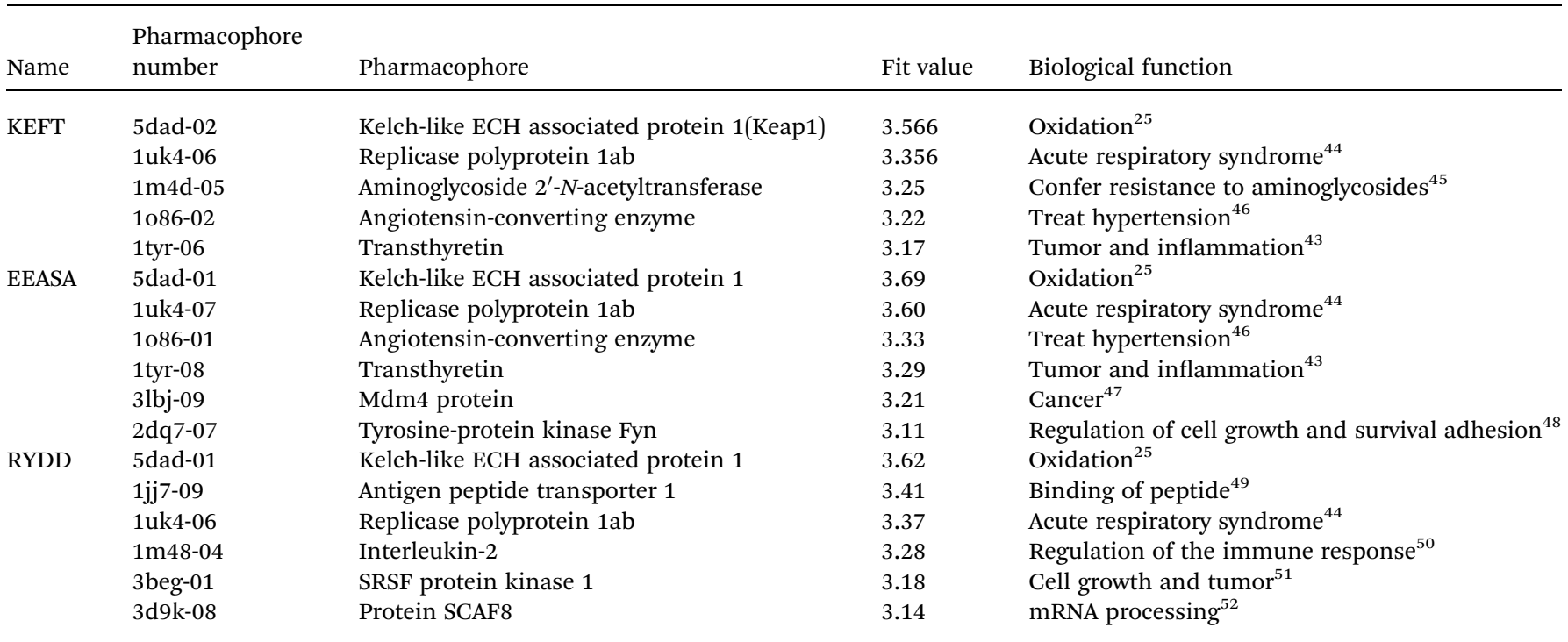

Table 3 The results of molecular docking

\begin{tabular}{|c|c|c|c|c|c|c|}
\hline Abbreviation & Name & Pose number & -CIE & $\begin{array}{l}\text { Number } \\
\text { of } \mathrm{HB}\end{array}$ & $\begin{array}{l}\text { Number of } \\
\pi \text {-bond }\end{array}$ & $\begin{array}{l}\text { Number of amino acid } \\
\text { residues interacting }\end{array}$ \\
\hline TX6 & $\begin{array}{l}\text { (6aS,7S,10aS)-8-hydroxy-4-methoxy- } \\
\text { 2,7,10a-trimethyl-5,6, } \\
\text { 6a,7,10,10a-hexahydrobenzo[h] } \\
\text { quinazoline-9-carbonitrile }\end{array}$ & 1 & 26.094 & - & 10 & 12 \\
\hline KEFT & Lys-Glu-Phe-Thr & 1 & 66.6412 & 5 & - & 14 \\
\hline
\end{tabular}

number of amino acid residues involved in hydrogen bonding (HB) and VDW is 3 and 6, respectively. KEFT interacts with Met147, Lys131 and Cys151 to form 5 HBs (Fig. 4E and F). As shown in Fig. $4 \mathrm{H}$ and $\mathrm{I}$, there are 12 amino acid residues associated with Keap1 and EEASA interactions, and 6 HBs interact with Lys131, Arg135, Met147, His129, Cys151 and Lys150 to participate in 6 VDW amino acid residues. There are 14 amino acid residues bound to RYDD and Keap1, among which 2 are HBs; these are Lys150 and Asp87, respectively. In addition, 4 amino acids are involved in VDW (Fig. $4 \mathrm{~K}$ and L). All of these results indicate that KEFT, EEASA and RYDD can bind to the Keap1 protein.

\section{Effect of antioxidant activity}

There was no significant change $(P>0.05)$ in the body weight of mice in the experimental group and control group (Fig. 5). Compared to the control group, the levels of serum TC $(2.83 \pm$ $\left.0.35 \mathrm{mmol} \mathrm{g}^{-1}, P<0.05\right)$, TG $\left(1.22 \pm 0.11 \mathrm{mmol} \mathrm{g}^{-1}, P<0.05\right)$ and LDL $\left(0.32 \pm 0.02 \mathrm{mmol} \mathrm{g}^{-1}, P<0.05\right)$ in the experiment group were significantly reduced, while the HDL level was increased (5.18 $\left.\pm 0.87 \mathrm{mmol} \mathrm{g}^{-1}, P<0.05\right)$. The situation in the liver is similar to that of the serum (Fig. 6), with reduced TC $\left(0.05 \pm 0.01 \mathrm{mmol} \mathrm{g}^{-1}, P<0.05\right), \mathrm{TG}\left(0.11 \pm 0.04 \mathrm{mmol} \mathrm{g}^{-1}, P<\right.$ $0.05)$, and LDL $\left(0.01 \pm 0.01 \mathrm{mmol} \mathrm{g}^{-1}, P<0.01\right)$ and increased HDL $\left(0.02 \pm 0.005 \mathrm{mmol} \mathrm{g}^{-1}, P<0.05\right)$.

Compared to the control group, the levels of serum GSH-Px $\left(893.11 \pm 206.58 \mathrm{U} \mathrm{ml}^{-1} / \mathrm{U} \mathrm{mg}^{-1}, P<0.05\right)$ and SOD $(86.29 \pm$ $\left.11.18 \mathrm{U} \mathrm{ml}^{-1} / \mathrm{U} \mathrm{mg}^{-1}, P<0.05\right)$ in the experimental group were significantly increased, while the MDA level $(6.19 \pm 1.38 \mathrm{nmol}$ $\left.\mathrm{ml}^{-1}, P<0.05\right)$ was significantly decreased. The situation in the liver is similar to that of the serum (Fig. 6), with increased GSH$\operatorname{Px}\left(831.15 \pm 88.92 \mathrm{U} \mathrm{ml}^{-1} / \mathrm{U} \mathrm{mg}^{-1}, P<0.05\right)$ and SOD $(202.96 \pm$ $\left.35.76 \mathrm{U} \mathrm{ml}^{-1} / \mathrm{U} \mathrm{mg}^{-1}, P<0.05\right)$ and decreased MDA levels $(1.72$ $\left.\pm 0.51 \mathrm{nmol} \mathrm{ml}^{-1}, P<0.05\right)$.

\section{Characterization of the mRNA expression level}

Keap1 is an important regulator of cellular oxidative stress. The $\gamma 2$ regulatory subunit of AMP-activated protein kinase, which is encoded by the gene Prkag2, is a heterotrimeric Ser/Thr kinase that acts against oxidative damage. ${ }^{26}$ To verify the antioxidation effect at the mRNA-level, two anti-oxidative related genes were selected for qRT-PCR analysis (Fig. 7). The results showed that Prkag2 was significantly up-regulated in the liver $(P<0.05)$ and 

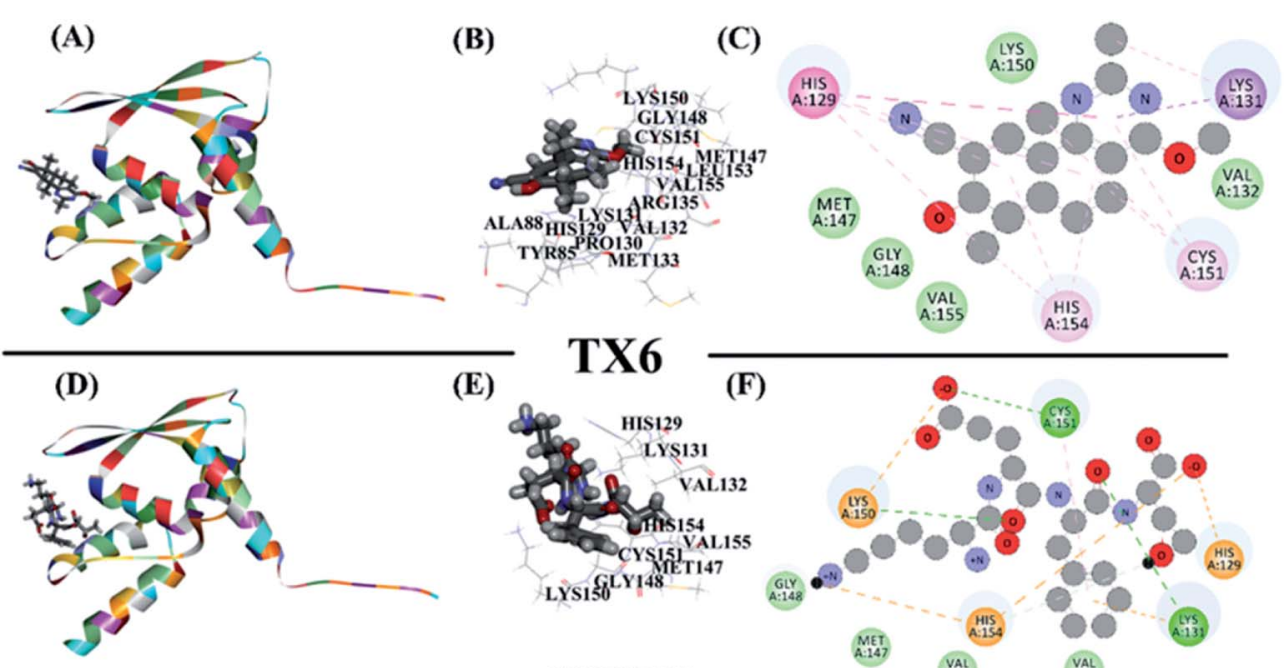

(E)
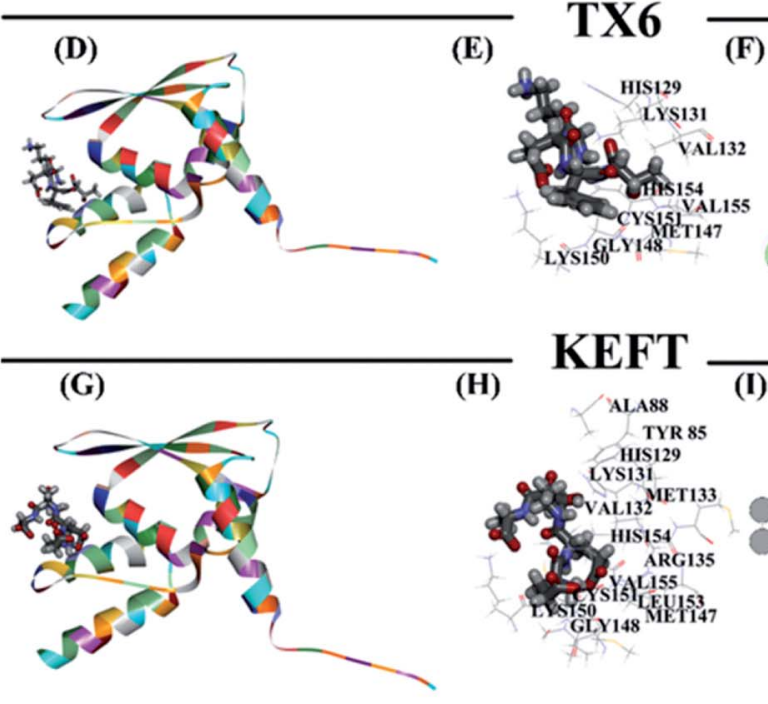

(J)

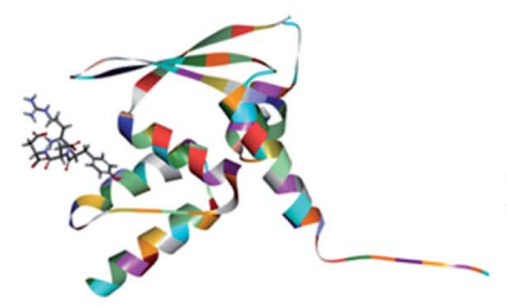

(H)

(K)

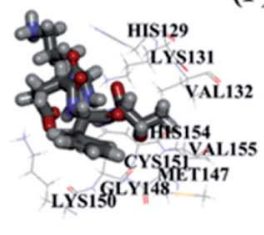

KEFT

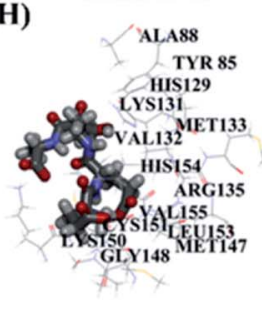

EEASA

(F)

(I)
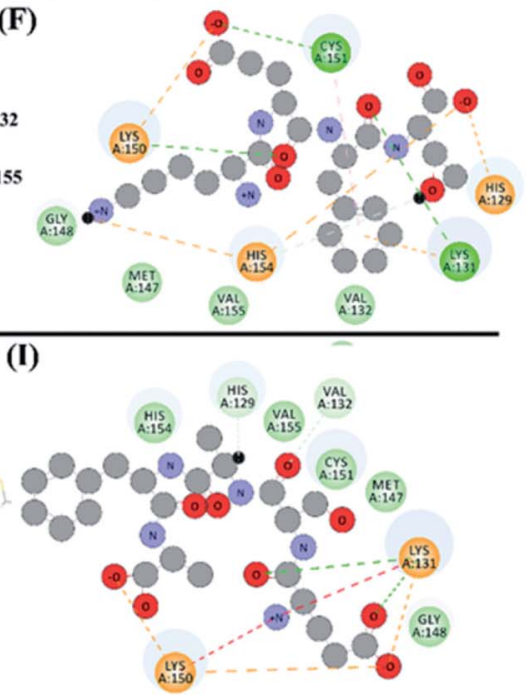

(L)
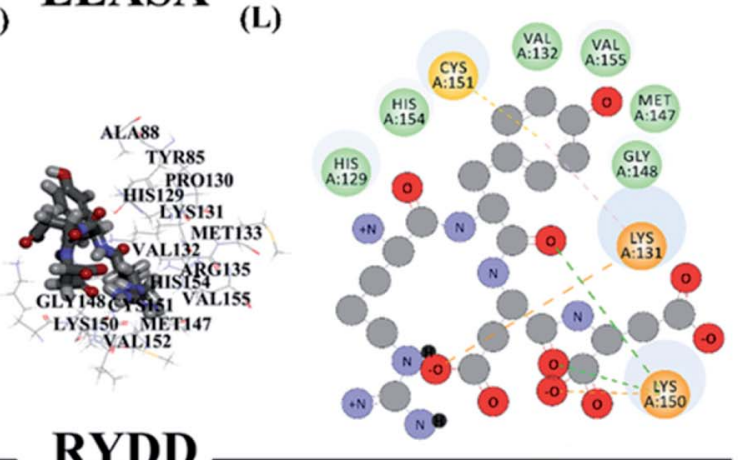

RYDD

Fig. 4 The interaction of polypeptides with Keap1. (A), (D), (G), and (J) the protein and polypeptide are integrally bound. The polypeptide is compatible with the protein active site. (B), (E), (H), and (K) the polypeptide binds at the active site. (C), (F), (I), and (L) amino acid residue 2D diagram.

brain $(P<0.05)$, whereas Keap1 was only significantly downregulated only in the brain $(P<0.01)$ after treatment.

\section{Discussion}

In this study, we firstly optimized the enzymatic hydrolysis conditions via traditional response surface experiments, and the results is shown in Fig. 1 and 2. The use of enzymatic hydrolysis is often considered as an appropriate and useful method for maintaining the nutritional value of proteins and improving the functional properties of them. ${ }^{27}$ The process depends on several factors including enzyme type, enzyme concentration, ratio of enzyme, solid-to-liquid ratio, temperature and enzymolysis time. ${ }^{28}$ These factors cooperatively affect the enzyme activity and thus make the hydrolysis process more controllable. Several enzymes, such as alkaline protease, trypsin, papain, pepsin, flavourzyme, animal protein hydrolase, and thermolysin, have been widely used in fish proteins hydrolyzing. ${ }^{29}$ In this experiment, the alkaline protease and trypsin were chosen via single factor experiment, and then BoxBehnken design (BBD), a popular tool to design response surface, was used to optimize the hydrolysis conditions. ${ }^{30-32}$

The peptides in the dark muscle hydrolysate were subsequently sequenced after enzymolysis, and the dominant polypeptides were predicted to bind to an antioxidant protein (Keap1) (Fig. 4, Tables 2 and 3). The subsequent animal experiments showed that MDA in the treatment group were significantly reduced and SOD and GSH-Px were significantly 


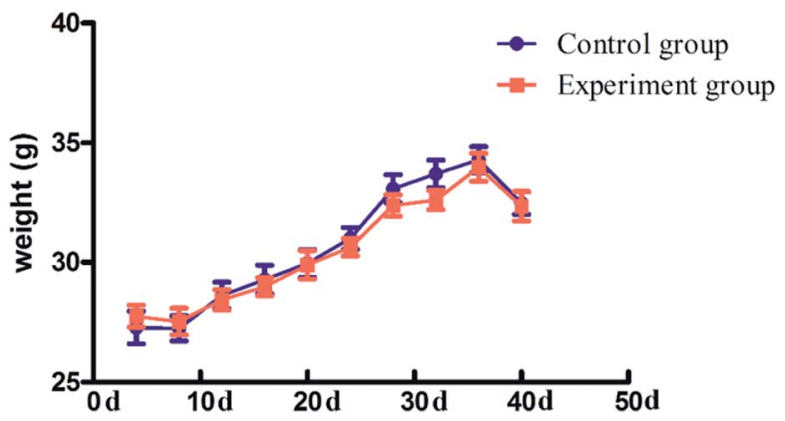

Fig. 5 The body weight of mice in the control group and experiment groups. The data are expressed as the mean $\pm S D, n=12$. ANOVA was used to assess the differences. ${ }^{*} P<0.05 ;{ }^{*} P<0.01$, compared with the control group.

increased (Fig. 6) compared to the control group. In addition, Keap1 was down-regulated in the treatment group, while Prkag2 was significantly up-regulated. All of these results indicate that the hydrolysates derived from tuna dark muscle are promising candidates for antioxidation and blood lipid control.

The research findings showed that the serum TC, TG and LDL-C levels were significantly decreased in the experimental group and that the serum HDL-C level was increased, and similar results were obtained in the liver. Oxidative stress has a negative effect on the body, which is induced by free radicals and is considered to be an important factor leading to diabetes mellitus and cardiovascular disease, causing a series of harmful biochemical reactions that lead to dyslipidemia. ${ }^{33}$ Various active substances not only act as an antioxidant but also regulate blood lipid metabolism. The Panax quinquefolius polysaccharide peptide showed an anti-oxidant effect and can be used to reduce blood glucose and blood lipid in diabetic mice. ${ }^{34}$ Goose oil improves the antioxidant capacity, ameliorates blood lipid metabolism and resists atherosclerosis in mice. ${ }^{35}$ Research has shown that $13.6 \%$ of dongchunghacho rice supplementation to polished rice has antioxidant potential and is hypocholesterolemic as well as hypoglycemic in STZ-induced diabetic rats. ${ }^{36}$ However, the link between oxidative stress and lipid metabolism is not clear. On the one hand, hydrolysate treatment significantly increased the activities of GSH-Px and SOD and decreased the MDA levels as well as returned blood lipid metabolism to normal via free radical scavenging. On the other hand, research has shown that the serum TG levels influence the plasma MDA concentrations. ${ }^{37}$ Thus, we hypothesize that TG can improve blood lipid disorders by increasing the body's antioxidant capacity. In addition, reverse docking results show that RYDD and KEFT can bind to the low-density lipoprotein receptor (2.66 and 2.63 fit-value, respectively). The low-density lipoprotein receptor, which can bind LDL, is the major cholesterol-carrying lipoprotein in plasma and transports cholesterol into cells by endocytosis to regulate hyperlipidemia (ESI Table S4†). LDL, known as "bad cholesterol", plays a role in transporting endogenous cholesterol and cholesteryl ester in plasma and its concentration is associated with an increased prevalence of atherosclerosis. Degradation of LDL occurs via the LDL receptor pathway. In this experiment, the binding of polypeptide to KEAP1 is relatively higher than to the low-density lipoprotein receptor, which prevents the binding of polypeptide to the LDL receptor. Therefore, the LDL receptor binds to LDL and LDL is degraded through the LDL receptor pathway, which may explain the lipid-lowering function.

Reverse docking is a powerful tool that is used for drug repositioning and rescue. ${ }^{10,38}$ From our results, we used reverse
A

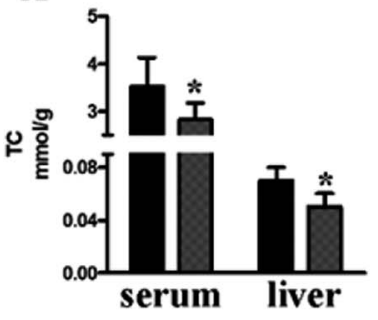

B

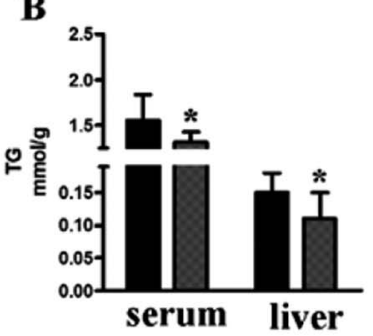

C

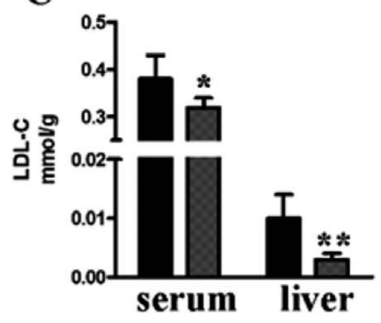

D

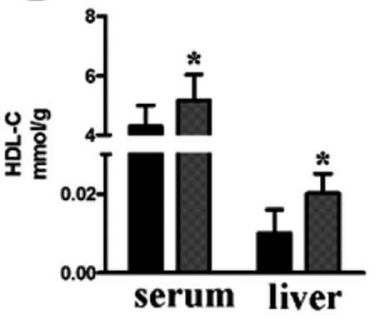

E

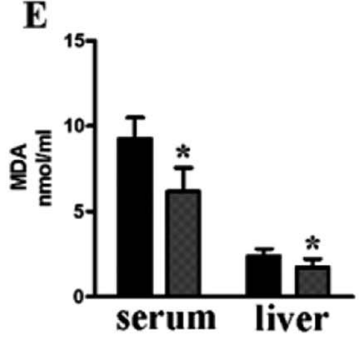

$\mathbf{F}$

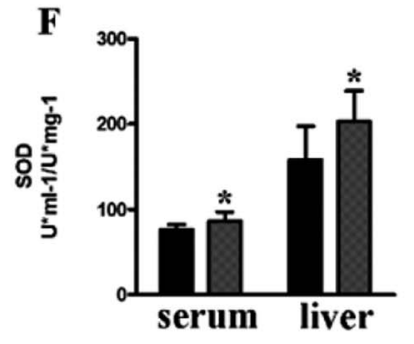

Control group

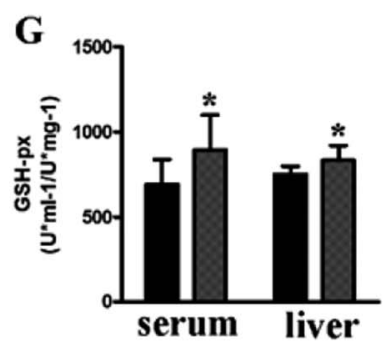

Experiment group

Fig. 6 The effects of the polypeptide on the physiological and biochemical indexes of mice. (A) The TC content (B) The TG content (C) the LDL-C content (D) the HDL-C content (E) the MDA content (F) the SOD activities (G) the GSH-Px activities; the data are expressed as the mean \pm SD, $n=$ 12. ANOVA was used to assess the differences, $* P<0.05 ; * *, P<0.01$, compared with the control group. 
A

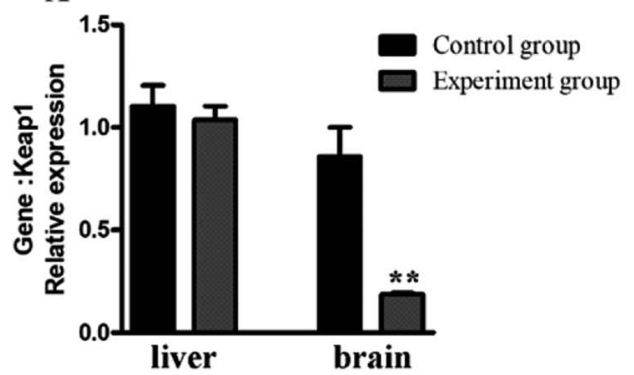

B

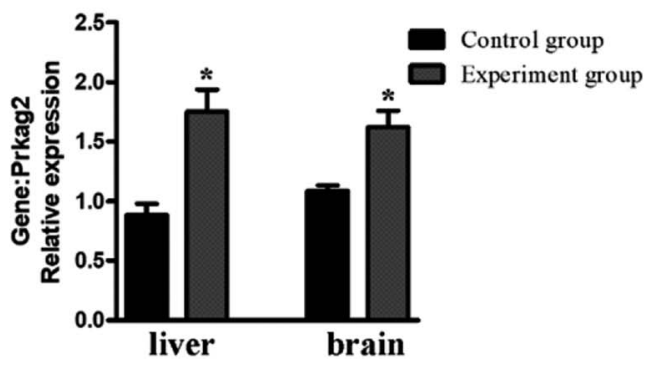

Fig. 7 Relative expression of antioxidant genes in the mouse liver and brain. $* P<0.05$; **, $P<0.01$, compared with the control group.

docking to find an antioxidant-related protein that can affect the oxidative stress system. In response to active oxygen damage, the body has formed a complex oxidative stress response system, the coordination response is regulated by the antioxidant responsive element (ARE) of these protective genes in the upstream regulation region..$^{39,40} \mathrm{Nrf} 2$ is a critical factor for regulating the intracellular expression of many antioxidants. ${ }^{41}$ Keapl-Nrf2/ARE, as an effective antioxidant regulation pathway, has played an important role in cellular defense and reduces the oxidative stress of the human body. ${ }^{42}$ Inhibition of Keap1 activity activates antioxidant stress of the Nrf2/ARE signaling pathway. In this study, the value of -CIE of KEFT, EEASA and RYDD were higher than that of TX6, which acted as a reference to evaluate the binding degree between polypeptides and the Keap1 protein, indicating better binding of KEFT, EEASA and LCGEC with Keap1 than TX6. Thus, the polypeptide can inhibit the activity of Keap1and activate the antioxidant stress of the Nrf2/ARE signaling pathway. In addition, Prkag2 was significantly increased in the polypeptide treatment group. Prkag2 is involved in the AMPK pathway, and AMPK is a heterotrimeric Ser/Thr kinase that acts against oxidative damage. Therefore, we believe that the antioxidant activity of hydrolyzate can be achieved by affecting multiple pathways.

From the reverse docking results, polypeptide functions were predicted, such as anti-hypertension, cell growth regulation, anti-tumor activity, and immune system regulation. In this study, KEFT and EEASA bind to 1086 (angiotensin-converting enzyme) (3.22 and 3.33 fit-value, respectively), which may reduce the formation of angiotensinIIand increase the activity of bradykinin. This function can effectively relieve high blood pressure, heart failure, diabetes, hypertension and other diseases. ${ }^{17}$ KEFT and EEASA also can bind to 1 tyr (transthyretin) (3.17 and 3.29 fit-value, respectively), which is an inhibitor of monocyte and endothelial cell interleukin-1 production; therefore, the combination of polypeptides and transthyretin can effectively promote interleukin-1 production and exhibit antitumor and immune regulation activity. ${ }^{43}$

In this study, the in silico test showed that the three peptides (KEFT, EEASA, RYDD) in the hydrolysate showed antioxidant activity via binding to protein Keap1, and the subsequently animal experiments confirmed the prediction. Therefore, it provides a high throughput strategy to screen new function of hydrolysate. However, due to a lack of the animal experiment with the synthesized single peptide (KEFT, EEASA, RYDD), it is hard to indicate whether they actually possess antioxidant activity, as well as which peptide play a key role in in vivo antioxidant activity. This shortage of the strategy needs to be considered in the further applications.

\section{Conflicts of interest}

The authors declare no competing financial interest.

\section{Acknowledgements}

This work was supported by the Regional Demonstration Project of Marine Economic Innovation and Development in 2014 and 2016, and the K. C. Wong Magna Fund in Ningbo University. We thank Nature Research Editing Service for English language editing.

\section{References}

1 B. Grigsby, H. Rodriguezrilo and K. Khan, Antioxidants and chronic pancreatitis: theory of oxidative stress and trials of antioxidant therapy, Dig. Dis. Sci., 2012, 57, 835-841.

$2 \mathrm{H}$. Sies, Hydrogen peroxide as a central redox signaling molecule in physiological oxidative stress: Oxidative eustress, Redox Biol., 2017, 11, 613-619.

3 S. Y. Jun, P. J. Park, W. K. Jung and S. K. Kim, Purification and characterization of an antioxidative peptide from enzymatic hydrolysate of yellowfin sole (Limanda aspera) frame protein, Eur. Food Res. Technol., 2004, 219, 20-26.

4 B. Mbatia, L. A. Ogonda, E. K. Muge and F. J. Mulaa, Antioxidative and functional properties of Rastrineobola argentea (Dagaa) fish protein hydrolysate, J. Sci. Food Agric., 2014, 41, 764-772.

5 H. Miao, Q. Liu, H. Bao, X. Wang and S. Miao, Effects of different freshness on the quality of cooked tuna steak, Innovative Food Sci. Emerging Technol., 2017, 44, 67-73.

6 J. Y. Je, Z. J. Qian, H. G. Byun and S. K. Kim, Purification and characterization of an antioxidant peptide obtained from tuna backbone protein by enzymatic hydrolysis, Process Biochem., 2007, 42, 840-846.

7 J. S. Hwang, Impact of processing on stability of angiotensin I-converting enzyme (ACE) inhibitory peptides obtained from tuna cooking juice, Food Res. Int., 2010, 43, 902-906. 
8 Q. Wang, J. He, D. Wu, J. Wang, J. Yan and H. Li, Interaction of $\alpha$-cyperone with human serum albumin: Determination of the binding site by using Discovery Studio and via spectroscopic methods, J. Lumin., 2015, 164, 81-85.

9 K. Park and A. E. Cho, Using Reverse Docking to Identify Potential Targets for Ginsenosides, J. Ginseng Res., 2017, 41(4), 534-539.

$10 \mathrm{~S}$. J. Chen and J. L. Ren, Identification of a potential anticancer target of danshensu by inverse docking, Asian Pacific Journal of Cancer Prevention, 2014, 15, 111-116.

11 B. B. Chen, T. A. Coon, J. R. Glasser, B. J. Mcverry, J. Zhao, Y. Zhao, C. Zou, B. Ellis, F. C. Sciurba and Y. Zhang, A combinatorial $\mathrm{F}$ box protein directed pathway controls TRAF adaptor stability to regulate inflammation, Nat. Immunol., 2013, 14, 470-479.

12 S. C. Ko, J. Jang, B. R. Ye, M. S. Kim, I. W. Choi, W. S. Park, S. J. Heo and W. K. Jung, Purification and molecular docking study of angiotensin I-converting enzyme (ACE) inhibitory peptides from hydrolysates of marine sponge Stylotella aurantium, Process Biochem., 2017, 54, 180-187.

13 K. Park and A. E. Cho, Using Reverse Docking to Identify Potential Targets for Ginsenosides, J. Ginseng Res., 2017, 41, 534-539.

14 G. Ulrike, K. Teresa, P. Florian, C. E. Mair, L. Thierry, S. Daniela and J. M. Rollinger, Accessing biological actions of Ganoderma secondary metabolites by in silico profiling, Phytochemistry, 2015, 114, 114-124.

15 M. Tu, C. Wang, C. Chen, R. Zhang, H. Liu, W. Lu, L. Jiang and $\mathrm{M}$. Du, Identification of a novel ACE-inhibitory peptide from casein and evaluation of the inhibitory mechanisms, Food Chem., 2018, 256, 98-104.

16 F. Charte, I. Romero, M. D. Pérez-Godoy, A. J. Rivera and E. Castro, Comparative analysis of data mining and response surface methodology predictive models for enzymatic hydrolysis of pretreated olive tree biomass, Comput. Chem. Eng., 2017, 101, 23-30.

17 J. Jaeyoung, L. Kahwa, L. Mihyun and A. Changbum, Antioxidant and antihypertensive protein hydrolysates produced from tuna liver by enzymatic hydrolysis, Food Res. Int., 2009, 42, 1266-1272.

18 F. Bittar, Z. Ouchenane, F. Smati, D. Raoult and J. M. Rolain, MALDI-TOF-MS for rapid detection of staphylococcal Panton-Valentine leukocidin, Int. J. Antimicrob. Agents, 2009, 34, 467.

19 Y. T. Zhang, Y. P. Geng, L. Zhou, B. C. Lai, L. S. Si and Y. L. Wang, Identification of proteins of human colorectal carcinoma cell line SW480 by two-dimensional electrophoresis and MALDI-TOF mass spectrometry, World J. Gastroenterol., 2005, 11, 4679-4684.

20 T. Dilger, H. Melzl and A. Gessner, Rapid and reliable identification of waterborne Legionella species by MALDITOF mass spectrometry, J. Microbiol. Methods, 2016, 127, 154-159.

21 E. J. Lee, J. Hur, A. H. Sun, Y. Jo, S. Y. Lee, M. J. Choi and G. S. Han, Fish collagen peptide inhibits the adipogenic differentiation of preadipocytes and ameliorates obesity in high fat diet-fed mice, Int. J. Biol. Macromol., 2017, 104, 281-286.

22 D. Zhang, J. Xu, W. He, Q. Tong, L. Chen, J. Zhou and X. Su, Characterization of Enterobacter cloacae under phoxim stress by two-dimensional gel electrophoresis, Biotechnol. Bioprocess Eng., 2015, 20, 403-409.

23 S. U. Morton, M. Joshi, T. Savic, A. H. Beggs and P. B. Agrawal, Skeletal muscle microRNA and messenger RNA profiling in cofilin-2 deficient mice reveals cell cycle dysregulation hindering muscle regeneration, PLoS One, 2015, 10, e0123829.

24 Q. Wu, J. Du, J. Jia and C. Kuang, Production of ACE inhibitory peptides from sweet sorghum grain protein using alcalase: Hydrolysis kinetic, purification and molecular docking study, Food Chem., 2016, 199, 140-149.

25 C. Huerta, X. Jiang, I. Trevino, C. F. Bender, D. A. Ferguson, B. Probst, K. K. Swinger, V. S. Stoll, P. J. Thomas and I. Dulubova, Characterization of novel small-molecule NRF2 activators: Structural and biochemical validation of stereospecific KEAP1 binding, Biochim. Biophys. Acta, Biomembr., 2016, 1860, 2537-2552.

26 A. Schlernitzauer, B. Chabi, G. Cross, G. Cabello, R. Magous and C. Wrutniak-Cabello, Chicoric acid is a potent antioxidant regulating the AMPK pathway, Ann. Nutr. Metab., 2011, 58, 50.

27 M. Chalamaiah, G. N. Rao, D. G. Rao and T. Jyothirmayi, Protein hydrolysates from meriga (Cirrhinus mrigala) egg and evaluation of their functional properties, Food Chem., 2010, 120, 652-657.

28 F. M. Diniz and A. M. Martin, Optimization of nitrogen recovery in the enzymatic hydrolysis of dogfish (Squalus acanthias) protein. Composition of the hydrolysates, Int. J. Food Sci. Nutr., 1997, 48, 191-200.

29 A. Noman, Y. Xu, W. Q. Al-Bukhaiti, S. M. Abed, A. H. Ali, A. H. Ramadhan and W. Xia, Influence of enzymatic hydrolysis conditions on the degree of hydrolysis and functional properties of protein hydrolysate obtained from Chinese sturgeon (Acipenser sinensis) by using papain enzyme, Process Biochem., 2018, 67, 19-28.

30 S. L. Ferreira, R. E. Bruns, H. S. Ferreira, G. D. Matos, J. M. David, G. C. Brandão, S. E. Da, L. A. Portugal, P. S. dos Reis and A. S. Souza, Box-Behnken design: An alternative for the optimization of analytical methods, Anal. Chim. Acta, 2007, 597, 179.

31 T. F. Meyabadi and F. Dadashian, Optimization of enzymatic hydrolysis of waste cotton fibers for nanoparticles production using response surface methodology, Fibers Polym., 2012, 13, 313-321.

32 R. G. Dadzie, H. Ma, E. E. Abano, W. Qu and S. Mao, Optimization of process conditions for production of angiotensin I-converting enzyme (ACE) inhibitory peptides from vital wheat gluten using response surface methodology, Food Sci. Biotechnol., 2013, 22, 1531-1537.

33 M. Matsuda and I. Shimomura, Increased oxidative stress in obesity: implications for metabolic syndrome, diabetes, hypertension, dyslipidemia, atherosclerosis, and cancer, Obesity Research \& Clinical Practice, 2013, 7, 330-341. 
34 R. Chen, D. Chen and J. Zhang, Lowering Blood Glucose,Blood Lipids and Antioxidant Effects of Panax quinquefolius Polysaccharide Peptide on Diabetic Mice, Acta Agric. Boreali-Occident. Sin., 2013, 22(11), 195-201.

35 B. Zhang, B. Wang, G. E. Wenhua, M. Zhang, B. Yue and L. U. Yanyan, Effects of different dose goose oils on blood lipid metabolism and antioxidant capacity of mice, China Oils Fats, 2012, 37, 31-35.

36 J. H. Park, N. S. Park, M. L. Sang and E. Park, Effect of Dongchunghacho rice on blood glucose level, lipid profile, and antioxidant metabolism in streptozotocin-induced diabetic rats, Food Sci. Biotechnol., 2011, 20, 933-940.

37 A. Ceriello, N. Bortolotti, E. Falleti, C. Taboga, L. Tonutti, A. Crescentini, E. Motz, S. Lizzio, A. Russo and E. Bartoli, Total radical-trapping antioxidant parameter in NIDDM patients, Diabetes Care, 1997, 20, 194-197.

38 G. Ulrike, K. Teresa, P. Florian, C. E. Mair, L. Thierry, S. Daniela and J. M. Rollinger, Accessing biological actions of Ganoderma secondary metabolites by in silico profiling, Phytochemistry, 2015, 114, 114.

39 K. Itoh, N. Wakabayashi, Y. Katoh, T. Ishii, T. O'Connor and M. Yamamoto, Keap1 regulates both cytoplasmic-nuclear shuttling and degradation of $\mathrm{Nrf} 2$ in response to electrophiles, Genes Cells, 2003, 8, 379.

40 T. H. Rushmore, M. R. Morton and C. B. Pickett, The antioxidant responsive element. Activation by oxidative stress and identification of the DNA consensus sequence required for functional activity, J. Biol. Chem., 1991, 266, 11632-11639.

41 D. Stewart, E. Killeen, R. Naquin, S. Alam and J. Alam, Degradation of transcription factor Nrf2 via the ubiquitinproteasome pathway and stabilization by cadmium, J. Biol. Chem., 2003, 278, 2396-2402.

42 J. A. Rubiolo, G. Mithieux and F. V. Vegaa, Resveratrol protects primary rat hepatocytes against oxidative stress damage:: Activation of the Nrf2 transcription factor and augmented activities of antioxidant enzymes, Eur. J. Pharmacol., 2008, 591, 66.

43 J. Mascali, L. Borish, S. Johnson, M. S. King, B. Coll and L. J. Rosenwasser, 269 Transthyretin is an inhibitor of monocyte and endothelial cell interleukin-1 production, Inflammation, 1992, 16, 471.
44 H. Yang, M. Yang, Y. Ding, Y. Liu, Z. Lou, Z. Zhou, L. Sun, L. Mo, S. Ye and H. Pang, The crystal structures of severe acute respiratory syndrome virus main protease and its complex with an inhibitor, P Natl A Sci, 2003, 100, 1319013195.

45 M. W. Vetting, S. S. Hegde, F. Javidmajd, J. S. Blanchard and S. L. Roderick, Aminoglycoside $2 \mid[$ prime $] \mid-\mathrm{N}-$ acetyltransferase from Mycobacterium tuberculosis in complex with coenzyme A and aminoglycoside substrates, Proc. Natl. Acad. Sci., 2002, 9, 653-658.

46 P. Sangsawad, S. Roytrakul and J. Yongsawatdigul, Angiotensin converting enzyme (ACE) inhibitory peptides derived from the simulated in vitro gastrointestinal digestion of cooked chicken breast, J. Funct. Foods, 2017, 29, 77-83.

47 G. M. Popowicz, A. Czarna, S. Wolf, K. Wang, W. Wang, A. Dömling and T. A. Holak, Structures of low molecular weight inhibitors bound to MDMX and MDM2 reveal new approaches for p53-MDMX/MDM2 antagonist drug discovery, Cell Cycle, 2010, 9, 1104-1111.

48 T. Kinoshita, M. Matsubara, H. Ishiguro, K. Okita and T. Tada, Structure of human Fyn kinase domain complexed with staurosporine, Biochem. Biophys. Res. Commun., 2006, 346, 840-844.

49 R. Gaudet and D. C. Wiley, Structure of the ABC ATPase domain of human TAP1, the transporter associated with antigen processing, EMBO J., 2001, 20, 4964-4972.

50 M. R. Arkin, M. Randal, W. L. Delano, J. Hyde, T. N. Luong, J. D. Oslob, D. R. Raphael, L. Taylor, J. Wang and R. S. Mcdowell, Binding of Small Molecules to an Adaptive Protein-Protein Interface, Proc. Natl. Acad. Sci. U.S.A, 2003, 100, 1603.

51 J. C. K. Ngo, K. Giang, S. Chakrabarti, C. T. Ma, N. Huynh, J. C. Hagopian, P. C. Dorrestein, X. D. Fu, J. A. Adams and G. Ghosh, A Sliding Docking Interaction Is Essential for Sequential and Processive Phosphorylation of an SR Protein by SRPK1, Mol. Cell, 2008, 29, 563-576.

52 R. Becker, B. Loll and A. Meinhart, Snapshots of the RNA processing factor SCAF8 bound to different phosphorylated forms of the carboxyl-terminal domain of RNA polymerase II, J. Biol. Chem., 2008, 283, 22659-22669. 